\title{
Ultimate physical limits to computation
}

\author{
Seth Lloyd \\ d'Arbeloff Laboratory for Information Systems and Technology \\ MIT Department of Mechanical Engineering \\ MIT 3-160, Cambridge, Mass. 02139 \\ slloyd@mit.edu
}

Computers are physical systems: what they can and cannot do is dictated by the laws of physics ${ }^{1-86}$. In particular, the speed with which a physical device can process information is limited by its energy ${ }^{11-26}$ and the amount of information that it can process is limited by the number of degrees of freedom it possesses ${ }^{5-40}$. This paper explores the physical limits of computation as determined by the speed of light $c$, the quantum scale $\hbar$ and the gravitational constant $G$. As an example, quantitative bounds are put to the computational power of an 'ultimate laptop' with a mass of one kilogram confined to a volume of one liter.

Over the past half century, the amount of information that computers are capable of processing and the rate at which they process it has doubled every two years, a phenomenon known as Moore's law. A variety of technologies - most recently, integrated circuits - have enabled this exponential increase in information processing power. There is no particular reason why Moore's law should continue to hold: it is a law of human ingenuity, not of nature. At some point, Moore's law will break down. The question is, When? Extrapolation of current exponential improvements over two more decades would result in computers that process information at the scale of individual atoms. Although an Avogadro scale computer that can act on $10^{23}$ bits might seem implausible, prototype quantum computers that store and process information on individual atoms have already been demonstrated ${ }^{64-65,76-80}$. Although existing quantum computers are small and simple, able to perform a few hundred operations on fewer than ten quantum bits or 'qubits,' the fact that they work at all indicates that there is nothing in the laws of physics that forbids the construction of an Avogadro-scale computer. 
The purpose of this article is to determine just what limits the laws of physics place on the power of computers. At first, this might seem a futile task: since we don't know the technologies by which computers one thousand, one hundred, or even ten years in the future will be constructed, how can we determine the physical limits of those technologies? In fact, as will now be shown, a great deal can be determined concerning the ultimate physical limits of computation simply from knowledge of the speed of light, $c=2.9979 \times 10^{8}$ meters per second, Planck's reduced constant, $\hbar=1.0545 \times 10^{-34}$ joule seconds, and the gravitational constant, $G=6.673 \times 10^{-11}$ meters cubed per kilogram second squared.

Boltzmann's constant, $k_{B}=1.3805 \times 10^{-23}$ joules per degree Kelvin, will also play a key role in translating between computational quantitites such as memory space and operations per bit per second, and thermodynamic quantitites such as entropy and temperature. This article reviews previous work on how physics limits the speed and memory of computers and presents new results: the derivation of the ultimate speed limit to computation, the trade-offs between memory and speed, and the analysis of the behavior of computers at physical extremes of high temperatures and densities are novel except as noted.

Before presenting methods for calculating these limits, it is important to note that there is no guarantee that these limits will ever be attained, no matter how ingenious computer designers become. Some extreme cases such as the black-hole computer described below are likely to prove extremely difficult or impossible to realize. Human ingenuity has proved great in the past, however, and before writing off physical limits as unattainable, one should realize that certain of these limits have already been attained within a circumscribed context in the construction of working quantum computers. The discussion below will note obstacles that must be sidestepped or overcome before various limits can be attained.

\section{Energy limits speed of computation}

To explore the physical limits of computation, let us calculate the ultimate computational capacity of a computer with a mass of one kilogram occupying a volume of one liter, roughly the size of a conventional laptop. Such a computer, operating at the limits of speed and memory space allowed by physics, will be called the 'ultimate laptop.'

First, ask what limits the laws of physics place on the speed of such a device. As will now be shown, to perform an elementary logical operation in time $\Delta t$ requires an average amount of energy $E \geq \pi \hbar / 2 \Delta t$. As a consequence, a system with average energy $E$ can perform a maximum of $2 E / \pi \hbar$ logical operations per second. A one kilogram computer has average energy $E=m c^{2}=8.9874 \times 10^{16}$ joules. Accordingly, the ultimate laptop can perform a maximimum of $5.4258 \times 10^{50}$ operations per second. 


\subsection{Maximum speed per logical operation}

For the sake of convenience, the ultimate laptop will be taken to be a digital computer. Computers that operate on non-binary or continuous variables obey similar limits to those that will be derived here. A digital computer performs computation by representing information in the terms of binary digits or bits, which can take the value 0 or 1 , and then processes that information by performing simple logical operations such as $A N D, N O T$ and FANOUT. The actual physical device that performs a logical operation is called a logic gate. The operation, $A N D$, for instance, takes two binary inputs $X$ and $Y$ and returns the output 1 if and only if both $X$ and $Y$ are 1; otherwise it returns the output 0 . Similarly, NOT takes a single binary input $X$ and returns the output 1 if $X=0$ and 0 if $X=1$. FANOUT takes a single binary input $X$ and returns two binary outputs, each equal to $X$. Any boolean function can be constructed by repeated application of $A N D, N O T$ and FANOUT. A set of operations that allows the construction of arbitrary boolean functions is called universal. The actual physical device that performs a logical operation is called a logic gate.

How fast can a digital computer perform a logical operation? During such an operation, the bits in the computer on which the operation is performed go from one state to another. The problem of how much energy is required for information processing was first investigated in the context of communications theory by Levitin ${ }^{11-16}$, Bremermann ${ }^{17-19}$, Beckenstein $^{20-22}$ and others, who showed that the laws of quantum mechanics determine the maximum rate at which a system with spread in energy $\Delta E$ can move from one distinguishable state to another. In particular, the correct interpretation of the time-energy Heisenberg uncertainty principle $\Delta E \Delta t \geq \hbar$ is not that it takes time $\Delta t$ to measure energy to an accuracy $\Delta E$ (a fallacy that was put to rest by Aharonov and Bohm ${ }^{23-24}$ ) but rather that that a quantum state with spread in energy $\Delta E$ takes time at least $\Delta t=\pi \hbar / 2 \Delta E$ to

evolve to an orthogonal (and hence distinguishable) state ${ }^{23-26}$. More recently, Margolus and Levitin ${ }^{15-16}$ extended this result to show that a quantum system with average energy $E$ takes time at least $\Delta t=\pi \hbar / 2 E$ to evolve to an orthogonal state.

\subsection{Performing quantum logic operations}

As an example, consider the operation NOT performed on a quantum bit or 'qubit' with logical states $|0\rangle$ and $|1\rangle$. (For readers unfamiliar with quantum mechanics, the 'bracket' notation $\mid>$ signifies that whatever is contained in the bracket is a quantummechanical variable; $|0\rangle$ and $|1\rangle$ are vectors in a two-dimensional vector space over the complex numbers.) To flip the qubit, one can apply a potential $H=E_{0}\left|E_{0}\right\rangle\left\langle E_{0}\left|+E_{1}\right| E_{1}\right\rangle\left\langle E_{1}\right|$ with energy eigenstates $\left|E_{0}\right\rangle=(1 / \sqrt{2})(|0\rangle+|1\rangle)$ and $\left|E_{1}\right\rangle=(1 / \sqrt{2})(|0\rangle-|1\rangle)$. Since 
$|0\rangle=(1 / \sqrt{2})\left(\left|E_{0}\right\rangle+\left|E_{1}\right\rangle\right)$ and $|1\rangle=(1 / \sqrt{2})\left(\left|E_{0}\right\rangle-\left|E_{1}\right\rangle\right)$, each logical state $|0\rangle,|1\rangle$ has spread in energy $\Delta E=\left(E_{1}-E_{0}\right) / 2$. It is easy to verify that after a length of time $\Delta t=\pi \hbar / 2 \Delta E$ the qubit evolves so that $|0\rangle \rightarrow|1\rangle$ and $|1\rangle \rightarrow|0\rangle$. That is, applying the potential effects a NOT operation in a time that attains the limit given by quantum mechanics. Note that the average energy $E$ of the qubit in course of the logical operation is $\langle 0|H| 0\rangle=\langle 1|H| 1\rangle=\left(E_{0}+E_{1}\right) / 2=E_{0}+\Delta E$. Taking the ground-state energy $E_{0}=0$ gives $E=\Delta E$. So the amount of time it takes to perform a $N O T$ can also be written $\Delta t=\pi \hbar / 2 E$. It is straightforward to show ${ }^{15-16}$ that no quantum system with average energy $E$ can move to an orthogonal state in a time less than $\Delta t$. That is, the speed with which a logical operation can be performed is limited not only by the spread in energy, but by the average energy. This result will prove to be a key component in deriving the speed limit for the ultimate laptop.

$A N D$ and FANOUT can be enacted in a way that is analogous to the NOT operation. A simple way to perform these operations in a quantum-mechanical context is to enact a so-called Toffoli or Controlled-Controlled-NOT operation ${ }^{31}$. This operation takes three binary inputs, $X, Y$, and $Z$ and returns three outputs, $X^{\prime}, Y^{\prime}$, and $Z^{\prime}$. The first two inputs pass through unchanged: $X^{\prime}=X, Y^{\prime}=Y$. The third input passes through unchanged unless both $X$ and $Y$ are 1, in which case it is flipped. This is universal in the sense that suitable choices of inputs allows the construction of AND, NOT, and FANOUT. When the third input is set to zero, $Z=0$, then the third output is the $A N D$ of the first two: $Z^{\prime}=X A N D Y$. So $A N D$ can be constructed. When the first two inputs are 1 , $X=Y=1$, the third output is the NOT of the third input, $Z^{\prime}=N O T Z$. Finally, when the second input is set to $1, Y=1$, and the third to zero, $Z=0$, the first and third output are the FANOUT of the first input, $X^{\prime}=X, Z^{\prime}=X$. So arbitrary boolean functions can be constructed from the Toffoli operation alone.

By embedding a Controlled-Controlled-NOT gate in a quantum context, it is straightforward to see that $A N D$ and FANOUT, like $N O T$, can be performed at a rate $2 E / \pi \hbar$ times per second, where $E$ is the average energy of the logic gate that performs the operation. More complicated logic operations that cycle through a larger number of quantum states (such as those on non-binary or continuous quantum variables) can be performed at a rate $E / \pi \hbar$ - half as fast as the simpler operations ${ }^{15-16}$. Existing quantum logic gates in optical-atomic and NMR quantum computers actually attain this limit. In the case of $N O T, E$ is the average energy of interaction of the qubit's dipole moment (electric dipole for optic-atomic qubits and nuclear magnetic dipole for NMR qubits) with the applied electromagnetic field. In the case of multi-qubit operations such as the Toffoli or simpler two bit Controlled-NOT operation, which flips the second bit if and only if the first bit 
is $1, E$ is the average energy in the interaction between the physical systems that register the qubits.

\subsection{Ultimate limits to speed of computation}

We are now in a position to derive our first physical limit to computation: energy limits speed. Suppose that one has a certain amount of energy $E$ to allocate to the logic gates of a computer. The more energy one allocates to a gate, the faster it can perform a logic operation. The total number of logic operations performed per second is equal to the sum over all logic gates of the operations per second per gate. That is, a computer can perform no more than

$$
\sum_{\ell} 1 / \Delta t_{\ell} \leq \sum_{\ell} 2 E_{\ell} / \pi \hbar=2 E / \pi \hbar
$$

operations per second. In other words, the rate at which a computer can compute is limited by its energy. (Similar limits have been proposed by Bremmerman in the context of the minimum energy required to communicate a bit ${ }^{17-19}$. These limits have been criticized, however, for misinterpreting the energy-time uncertainty relation ${ }^{21}$, and for failing to take into account the role of degeneracy of energy eigenvalues ${ }^{13-14}$ and the role of nonlinearity in communications ${ }^{7-9}$.) Applying this result to a one kilogram computer with energy $E=m c^{2}=8.9874 \times 10^{16}$ joules show that our ultimate laptop can perform a maximum of $5.4258 \times 10^{50}$ operations per second.

\subsection{Parallel and serial operation}

An interesting feature of this limit is that it is independent of computer architecture. One might have thought that a computer could be sped up by parallelization, i.e., by taking the energy and dividing it up amongst a large number of subsystems computing in parallel.

This is not the case: if one spreads the energy $E$ amongst $N$ logic gates, each one operates at a rate $2 E / \pi \hbar N$. The total number of operations per second, $N 2 E / \pi \hbar N=2 E / \pi \hbar$, remains the same. If the energy is allocated to fewer logic gates (more serial operation), the rate $1 / \Delta t_{\ell}$ at which they operate and the spread in energy per gate $\Delta E_{\ell}$ go up. If the energy is allocated to more logic gates (more parallel operation) then the rate at which they operate and the spread in energy per gate go down. Note that in this parallel case, the overall spread in energy of the computer as a whole is considerably smaller than the average energy: in general $\Delta E=\sqrt{\sum_{\ell} \Delta E_{\ell}^{2}} \approx \sqrt{N} \Delta E_{\ell}$ while $E=\sum E_{\ell} \approx N E_{\ell}$. Parallelization can help perform certain computations more efficiently, but it does not alter the total number of operations per second. As will be seen below, the degree of parallelizability of the computation to be performed determines the most efficient distribution of energy among 
the parts of the computer. Computers in which energy is relatively evenly distributed over a larger volume are better suited for performing parallel computations. More compact computers and computers with an uneven distribution of energy are better for performing serial computations.

\subsection{Comparison with existing computers}

Conventional laptops operate much more slowly than the ultimate laptop. There are two reasons for this inefficiency. First, most of the energy is locked up in the mass of the particles of which the computer is constructed, leaving only an infinitesimal fraction for performing logic. Second, a conventional computer employs many degrees of freedom (billions and billions of electrons) for registering a single bit. From the physical perspective, such a computer operates in a highly redundant fashion. There are good technological reasons for such redundancy: conventional designs rely on redundancy for reliability and manufacturability. In the present discussion, however, the subject is not what computers are but what they might be. The laws of physics do not require redundancy to perform logical operations: recently constructed quantum microcomputers use one quantum degree of freedom for each bit and operate at the Heisenberg limit $\Delta t=\pi \hbar / 2 \Delta E$ for the time needed to flip a bit ${ }^{64-65,76-80}$. Redundancy is required for error correction, however, as will be discussed below.

In sum, quantum mechanics provides a simple answer to the question of how fast information can be processed using a given amount of energy. Now it will be shown that thermodynamics and statistical mechanics provide a fundamental limit to how many bits of information can be processed using a given amount of energy confined to a given volume. Available energy necessarily limits the rate at which computer can process information. Similarly, the maximum entropy of a physical system determines the amount of information it can process. Energy limits speed. Entropy limits memory.

\section{Entropy limits memory space}

The amount of information that a physical system can store and process is related to the number of distinct physical states accessible to the system. A collection of $m$ two-state systems has $2^{m}$ accessible states and can register $m$ bits of information. In general, a system with $N$ accessible states can register $\log _{2} N$ bits of information. But it has been known for more than a century that the number of accessible states of a physical system, $W$, is related to its thermodynamic entropy by the formula: $S=k_{B} \ln W$, where $k_{B}$ is Boltzmann's constant. (Although this formula is inscribed on Boltzmann's tomb, it is originally due to Planck: before the turn of the century, $k_{B}$ was often known as Planck's constant.) 
The amount of information that can be registered by a physical system is $I=$ $S(E) / k_{B} \ln 2$, where $S(E)$ is the thermodynamic entropy of a system with expectation value for the energy $E$. Combining this formula with the formula $2 E / \pi \hbar$ for the number of logical operations that can be performed per second, we see that when it is using all its memory, the number of operations per bit per second that our ultimate laptop can perform is $k_{B} 2 \ln 2 E / \pi \hbar S \propto k_{B} T / \hbar$, where $T=(\partial S / \partial E)^{-1}$ is the temperature of a kilogram of matter in a maximum entropy in a liter volume. The entropy governs the amount of information the system can register and the temperature governs the number of operations per bit per second it can perform.

Since thermodynamic entropy effectively counts the number of bits available to a physical system, the following derivation of the memory space available to the ultimate laptop is based on a thermodynamic treatment of a kilogram of matter confined to a liter volume, in a maximum entropy state. Throughout this derivation, it is important to keep in mind that although the memory space available to the computer is given by the entropy of its thermal equilibrium state, the actual state of the ultimate laptop as it performs a computation is completely determined, so that its entropy remains always equal to zero. As above, we assume that we have complete control over the actual state of the ultimate laptop, and are able to guide it through its logical steps while insulating it from all uncontrolled degrees of freedom. As the following discussion will make clear, such complete control will be difficult to attain.

\subsection{Entropy, energy, and temperature}

In order to calculate the number of operations per second that could be performed by our ultimate laptop, we assumed that the expectation value of the energy was $E$. Accordingly, the total number of bits of memory space available to the computer is $S(E, V) / k_{B} \ln 2$, where $S(E, V)$ is the thermodynamic entropy of a system with expectation value of the energy $E$ confined to volume $V$. The entropy of a closed system is normally given by the so-called microcanonical ensemble, which fixes both the average energy and the spread in energy $\Delta E$, and assigns equal probability to all states of the system within a range $[E, E+\Delta E]$. In the case of the ultimate laptop, however, we wish to fix only the average energy, while letting the spread in energy vary according to whether the computer is to be more serial (fewer, faster gates, larger spread in energy) or parallel (more, slower gates, smaller spread in energy). Accordingly, the ensemble that should be used to calculate the thermodynamic entropy and the memory space available is the canonical ensemble, which maximizes $S$ for fixed average energy with no constraint on the spread in energy $\Delta E$. The canonical ensemble tells how many bits of memory are available 
for all possible ways of programming the computer while keeping its average energy equal to $E$. In any given computation with average energy $E$ the ultimate laptop will be in a pure state with some fixed spread of energy, and will explore only a small fraction of its memory space.

In the canonical ensemble a state with energy $E_{i}$ has probability $p_{i}=(1 / Z(T)) e^{-E_{i} / k_{B} T}$ where $Z(T)=\sum_{i} e^{-E_{i} / k_{B} T}$ is the partition function, and the temperature $T$ is chosen so that $E=\sum_{i} p_{i} E_{i}$. The entropy is $S=-k_{B} \sum_{i} p_{i} \ln p_{i}=E / T+k_{B} \ln Z$. The number of bits of memory space available to the computer is $S / k_{B} \ln 2$. The difference between the entropy as calculated using the canonical ensemble and that calculated using the microcanonical ensemble is minimal. There is some subtlety involved in using the canonical ensemble rather than the more traditional microcanonical ensemble, however. The canonical ensemble is normally used for open systems that interact with a thermal bath at temperature $T$. In the case of the ultimate laptop, however, it is applied to a closed system to find the maximum entropy given a fixed expectation value for the energy. As a result, the temperature $T=(\partial S / \partial E)^{-1}$ plays a somewhat different role in the context of physical limits of computation than it does in the case of an ordinary thermodynamic system interacting with a thermal bath. Integrating the relationship $T=(\partial S / \partial E)^{-1}$ over $E$ yields $T=C E / S$, where $C$ is a constant of order unity (e.g., $C=4 / 3$ for black-body radiation, $C=3 / 2$ for an ideal gas, and $C=1 / 2$ for a black hole). Accordingly, the temperature governs the number of operations per bit per second, $k_{B} \ln 2 E / \hbar S \approx k_{B} T / \hbar$, that a system can perform. As will become clear, the relationship between temperature and operations per bit per second is useful in investigating computation under extreme physical conditions.

(Box: The role of thermodynamics in computation. The fact that entropy and information are intimately linked has been known since Maxwell introduced his famous 'demon' well over a century ago ${ }^{1}$. Maxwell's demon is an hypothetical being that uses its information-processing ability to reduce the entropy of a gas. The first results in physics of information processing were derived in attempts to understand how Maxwell's demon could function $^{1-4}$. The role of thermodynamics in computation has been repeatedly examined over the last half century. In the 1950 's, von Neumann ${ }^{10}$ speculated that each logical operation performed in a computer at temperature $T$ must dissipate energy $k_{B} T \ln 2$, thereby increasing entropy by $k_{B} \ln 2$. This speculation proved to be false. The precise, correct statement of the role of entropy in computation was due to Landauer ${ }^{5}$, who showed that reversible, i.e. one-to-one, logical operations such as NOT can be performed without dissipation in principle, but that irreversible, many-to-one operations such as $A N D$ or 
$E R A S E$ require dissipation at least $k_{B} \ln 2$ for each bit of information lost. ( $E R A S E$ is a one-bit logical operation that takes a bit, 0 or 1, and restores it to 0 .) The argument behind Landauer's principle can be readily understood ${ }^{37}$. Essentially, the one-to-one dynamics of Hamiltonian systems implies that when a bit is erased the information that it contains has to go somewhere. If the information goes into observable degrees of freedom of the computer, such as another bit, then it has not been erased but merely moved; but if it goes into unobservable degrees of freedom such as the microscopic motion of molecules it results in an increase of entropy of at least $k_{B} \ln 2$.

In 1973, Bennett ${ }^{28-30}$ showed that all computations could be performed using reversible logical operations only. Consequently, by Landauer's principle, computation does not require dissipation. (Earlier work by Lecerf ${ }^{27}$ had anticipated the possibility of reversible computation, but not its physical implications. Reversible computation was discovered independently by Fredkin and Toffoli ${ }^{31}$.) The energy used to perform a logical operation can be 'borrowed' from a store of free energy such as a battery, 'invested' in the logic gate that performs the operation, and returned to storage after the operation has been performed, with a net 'profit' in the form of processed information. Electronic circuits based on reversible logic have been built and exhibit considerable reductions in dissipation over conventional reversible circuits ${ }^{33-35}$.

Under many circumstances it may prove useful to perform irreversible operations such as erasure. If our computer is subject to an error rate of $\epsilon$ bits per second, for example, then error-correcting codes can be used to detect those errors and reject them to the environment at a dissipative cost of $\epsilon k_{B} T_{E} \ln 2$ joules per second, where $T_{E}$ is the temperature of the environment. ( $k_{B} T \ln 2$ is the minimal amount of energy required to send a bit down an information channel with noise temperature $T .{ }^{14}$ ) Such error-correcting routines in our ultimate computer function as working analogs of Maxwell's demon, getting information and using it to reduce entropy at an exchange rate of $k_{B} T \ln 2$ joules per bit. In principle, computation does not require dissipation. In practice, however, any computer - even our ultimate laptop - will dissipate energy.

The ultimate laptop must reject errors to the environment at a high rate to maintain reliable operation. To estimate the rate at which it can reject errors to the environment, assume that the computer encodes erroneous bits in the form of black-body radiation at the characteristic temperature $5.87 \times 10^{8} \mathrm{~K}$ of the computer's memory. ${ }^{21}$ The StefanBoltzmann law for black-body radiation then implies that the number of bits per unit area than can be sent out to the environment is $\mathcal{B}=\pi^{2} k_{B}^{3} T^{3} / 60 \ln 2 \hbar^{3} c^{2}=7.195 \times 10^{42}$ bits per meter $^{2}$ per second. Since the ultimate laptop has a surface area of $10^{-2}$ square meters and is performing $\approx 10^{50}$ operations per second, it must have an error rate of less than $10^{-10}$ 
per operation in order to avoid over-heating. Even if it achieves such an error rate, it must have an energy throughput (free energy in and thermal energy out) of $4.04 \times 10^{26}$ watts turning over its own rest mass energy of $m c^{2} \approx 10^{17}$ joules in a nanosecond! The thermal load of correcting large numbers of errors clearly suggests the necessity of operating at a slower speed than the maximum allowed by the laws of physics. )

\subsection{Calculating the maximum memory space}

To calculate exactly the maximum entropy for a kilogram of matter in a liter volume would require complete knowledge of the dynamics of elementary particles, quantum gravity, etc. We do not possess such knowledge. However, the maximum entropy can readily be estimated by a method reminiscent of that used to calculate thermodynamic quantities in the early universe ${ }^{87}$. The idea is simple: model the volume occupied by the computer as a collection of modes of elementary particles with total average energy $E$. The maximum entropy is obtained by calculating the canonical ensemble over the modes. Here, we supply a simple derivation of the maximum memory space available to the ultimate laptop. A more detailed discussion of how to calculate the maximum amount of information that can be stored in a physical system can be found in the work of Bekenstein ${ }^{19-21}$.

For this calculation, assume that the only conserved quantities other than the computer's energy are angular momentum and electric charge, which we take to be zero. (One might also ask that baryon number be conserved, but as will be seen below, one of the processes that could take place within the computer is black hole formation and evaporation, which does not conserve baryon number.) At a particular temperature $T$, the entropy is dominated by the contributions from particles with mass less than $k_{B} T / 2 c^{2}$. The $\ell^{\prime}$ th such species of particle contributes energy $E=r_{\ell} \pi^{2} V\left(k_{B} T\right)^{4} / 30 \hbar^{3} c^{3}$ and entropy $S=2 r_{\ell} k_{B} \pi^{2} V\left(k_{B} T\right)^{3} / 45 \hbar^{3} c^{3}=4 E / 3 T$ where $r_{\ell}$ is equal to the number of particles/antiparticles in the species (i.e., 1 for photons, 2 for electrons/positrons) times the number of polarizations ( 2 for photons, 2 for electrons/positrons) times a factor that reflects particle statistics ( 1 for bosons, $7 / 8$ for fermions). As the formula for $S$ in terms of $T$ shows, each species contributes $(2 \pi)^{5} r_{\ell} / 90 \ln 2 \approx 10^{2}$ bits of memory space per cubic thermal wavelength $\lambda_{T}^{3}$ where $\lambda_{T}=2 \pi \hbar c / k_{B} T$. Re-expressing the formula for entropy as a function of energy, our estimate for the maximum entropy is

$$
S=(4 / 3) k_{B}\left(\pi^{2} r V / 30 \hbar^{3} c^{3}\right)^{1 / 4} E^{3 / 4}=k_{B} \ln 2 I
$$

where $r=\sum_{\ell} r_{\ell}$. Note that $S$ depends only insensitively on the total number of species with mass less than $k_{B} T / 2 c^{2}$. 
A lower bound on the entropy can be obtained by assuming that energy and entropy are dominated by black body radiation consisting of photons. In this case, $r=2$, and for a one kilogram computer confined to a volume of a liter we have $k_{B} T=8.10 \times 10^{-15}$ joules, or $T=5.87 \times 10^{8} \mathrm{~K}$. The entropy is $S=2.04 \times 10^{8}$ joule $/ \mathrm{K}$, which corresponds to an amount of available memory space $I=S / k_{B} \ln 2=2.13 \times 10^{31}$ bits. When the ultimate laptop is using all its memory space it can perform $2 \ln 2 k_{B} E / \pi \hbar S=3 \ln 2 k_{B} T / 2 \pi \hbar \approx 10^{19}$ operations per bit per second. As the number of operations per second $2 E / \pi \hbar$ is independent of the number of bits available, the number of operations per bit per second can be increased by using a smaller number of bits. In keeping with the prescription that the ultimate laptop operates at the absolute limits given by physics, in what follows, we assume that all available bits are used.

This estimate for the maximum entropy could be improved (and slightly increased) by adding more species of massless particles (neutrinos and gravitons) and by taking into effect the presence of electrons and positrons. Note that $k_{B} T / 2 c^{2}=4.51 \times 10^{-32}$ kilograms, compared with the electron mass of $9.1 \times 10^{-31}$ kilograms. That is, our kilogram computer in a liter is close to a phase transition at which electrons and positrons are produced thermally. A more exact estimate of the maximum entropy and hence the available memory space would be straightforward to perform, but the details of such a calculation would detract from our general exposition, and could only serve to alter $S$ slightly. $S$ depends insensitively on the number of species of effectively massless particles: a change of $r$ by a factor of 10,000 serves only to increase $S$ by a factor of 10 .

\subsection{Comparison with current computers}

The amount of information that can be stored by the ultimate laptop, $\approx 10^{31} \mathrm{bits}$, is much higher than the $\approx 10^{10}$ bits stored on current laptops. This is because conventional laptops use many degrees of freedom to store a bit where the ultimate laptop uses just one. There are considerable advantages to using many degrees of freedom to store information, stability and controllability being perhaps the most important. Indeed, as the above calculation indicates, in order to take full advantage of the memory space available, the ultimate laptop must turn all its matter into energy. A typical state of the ultimate laptop's memory looks like a plasma at a billion degrees Kelvin: the laptop's memory looks like a thermonuclear explosion or a little piece of the Big Bang! Clearly, packaging issues alone make it unlikely that this limit can be obtained, even setting aside the difficulties of stability and control.

Even though the ultimate physical limit to how much information can be stored in a kilogram of matter in a liter volume is unlikely to be attained, it may nonetheless be 
possible to get a fair way along the road to such bit densities. In other words, the ultimate limits to memory space may prove easier to approach than the ultimate limits to speed. Following Moore's law, the density of bits in a computer has gone down from approximately one per $\mathrm{cm}^{2}$ fifty years ago to one per $\mu \mathrm{m}^{2}$ today, an improvement of a factor of $10^{8}$. It is not inconceivable that a similar improvement is possible over the course of the next fifty years. In particular, there is no physical reason why it should not be possible to store one bit of information per atom. Indeed, existing NMR and ion-trap quantum computers already store information on individual nuclei and atoms (typically in the states of individual nuclear spins or in hyperfine atomic states). Solid-state NMR with high gradient fields or quantum optical techniques such as spectral hole-burning provide potential technologies for storing large quantities of information at the atomic scale. A kilogram of ordinary matter holds on the order of $10^{25}$ nuclei. If a substantial fraction of these nuclei can be made to register a bit, then one can get quite close to the ultimate physical limit of memory without having to resort to thermonuclear explosions. If, in addition, one uses the natural electromagnetic interactions between nuclei and electrons in the matter to perform logical operations, one is limited to a rate of approximately $10^{15}$ operations per bit per second,

yielding an overall information processing rate of $\approx 10^{40}$ operations per second in ordinary matter. Although less than the $\approx 10^{51}$ operations per second in the ultimate laptop, the maximum information processing rate in 'ordinary matter' is still quite respectable. Of course, even though such an 'ordinary matter' ultimate computer need not operate at nuclear energy levels, other problems remain: for example, the high number of bits still suggests substantial input/output problems. At an input/output rate of $10^{12}$ bits per second, an Avogadro-scale computer with $10^{23}$ bits would take about 10,000 years to perform a serial read/write operation on the entire memory. Higher throughput and parallel input/output schemes are clearly required to take advantage of the entire memory space that physics makes available.

\section{Size limits parallelization}

Up until this point, we have assumed that our computer occupies a volume of a liter. The previous discussion, however, indicates that benefits are to be obtained by varying the volume to which the computer is confined. Generally speaking, if the computation to be performed is highly parallelizable or requires many bits of memory, the volume of the computer should be greater and the energy available to perform the computation should be spread out evenly amongst the different parts of the computer. Conversely, if the computation to be performed is highly serial and requires fewer bits of memory, the energy should be concentrated in particular parts of the computer. 
A good measure of the degree of parallelization in a computer is the ratio between time it takes to communicate from one side of the computer to the other, and the average time it takes to perform a logical operation. The amount of time it takes to send a message from one side of a computer of radius $R$ to the other is $t_{\text {com }}=2 R / c$. The average time it takes a bit to flip in the ultimate laptop is the inverse of the number of operations per bit per second calculated above: $t_{\text {flip }}=\pi \hbar S / k_{B} 2 \ln 2 E$. Our measure of the degree of parallelization in the ultimate laptop is then

$$
t_{\text {com }} / t_{\text {flip }}=k_{B} 4 \ln 2 R E / \pi \hbar c S \propto k_{B} R T / \hbar c=2 \pi R / \lambda_{T} .
$$

That is, the amount of time it takes to communicate from one side of the computer to the other, divided by the amount of time it takes to flip a bit, is approximately equal to the ratio between the size of the system and its thermal wavelength. For the ultimate laptop, with $2 R=10^{-1}$ meters, $2 E / \pi \hbar \approx 10^{51}$ operations per second, and $S / k_{B} \ln 2 \approx 10^{31}$ bits, $t_{\text {com }} / t_{\text {flip }} \approx 10^{10}$. The ultimate laptop is highly parallel. A greater degree of serial computation can be obtained at the cost of decreasing memory space by compressing the size of the computer or making the distribution of energy more uneven. As ordinary matter obeys the Beckenstein bound ${ }^{20-22}, k_{B} R E / \hbar c S>1 / 2 \pi$, however, as one compresses the computer $t_{\text {com }} / t_{\text {flip }} \approx k_{B} R E / \hbar c S$ will remain greater than one: i.e., the operation will still be somewhat parallel. Only at the ultimate limit of compression - a black hole - is the computation entirely serial.

\subsection{Compressing the computer allows more serial computation}

Suppose that one wants to perform a highly serial computation on few bits. Then it is advantageous to compress the size of the computer so that it takes less time to send signals from one side of the computer to the other at the speed of light. As the computer gets smaller, keeping the energy fixed, the energy density inside the computer goes up. As the energy density in the computer goes up, different regimes in high energy physics are necessarily explored in the course of the computation. First the weak unification scale is reached, then the grand unification scale. Finally, as the linear size of the computer approaches its Schwarzchild radius, the Planck scale is reached. (No known technology could possibly achieve such compression.) At the Planck scale, gravitational effects and quantum effects are both important: the Compton wavelength of a particle of mass $m$, $\lambda_{C}=2 \pi \hbar / m c$ is on the order of its Schwarzschild radius, $2 G m / c^{2}$. In other words, to describe behavior at length scales of the size $\ell_{P}=\sqrt{\hbar G / c^{3}}=1.616 \times 10^{-35}$ meter, time scales $t_{P}=\sqrt{\hbar G / c^{5}}=5.391 \times 10^{-44}$ second, and mass scales of $m_{P}=\sqrt{\hbar c / G}=$ $2.177 \times 10^{-8}$ kilograms, a unified theory of quantum gravity is required. We do not currently 
possess such a theory. Nonetheless, although we do not know the exact number of bits that can be registered by a one kilogram computer confined to a volume of a liter, we do know the exact number of bits that can be registered by a one kilogram computer that has been compressed to the size of a black hole ${ }^{90}$. This is because the entropy of a black hole has a well-defined value.

In the following discussion, we use the properties of black holes to place limits on the speed, memory space, and degree of serial computation that could be approached by compressing a computer to the smallest possible size. Whether or not these limits could be attained, even in principle, is a question whose answer will have to await a unified theory of quantum gravity. (See Box 2)

The Schwarzschild radius of a 1 kilogram computer is $R_{S}=2 \mathrm{Gm} / \mathrm{c}^{2}=1.485 \times 10^{-27}$ meters. The entropy of a black hole is Boltzmann's constant times its area divided by 4 , as measured in Planck units. Accordingly, the amount of information that can be stored in a black hole is $I=4 \pi G m^{2} / \ln 2 \hbar c=4 \pi m^{2} / \ln 2 m_{P}^{2}$. The amount of information that can be stored by the 1 kilogram computer in the black-hole limit is $3.827 \times 10^{16}$ bits. A computer compressed to the size of a black hole can perform $5.4258 \times 10^{50}$ operations per second, the same as the 1 liter computer.

In a computer that has been compressed to its Schwarzschild radius, the energy per bit is $E / I=m c^{2} / I=\ln 2 \hbar c^{3} / 4 \pi m G=\ln 2 k_{B} T / 2$, where $T=(\partial S / \partial E)^{-1}=\hbar c / 4 \pi k_{B} R_{S}$ is the temperature of the Hawking radiation emitted by the hole. As a result, the time it takes to flip a bit on average is $t_{\text {flip }}=\pi \hbar I / 2 E=\pi^{2} R_{S} / c \ln 2$. In other words, according to a distant observer, the amount of time it takes to flip a bit, $t_{\text {flip }}$, is on the same order as the amount of time $t_{\text {com }}=\pi R_{S} / c$ it takes to communicate from one side of the hole to the other by going around the horizon: $t_{\text {com }} / t_{\text {flip }}=\ln 2 / \pi$. In contrast to computation at lesser densities, which is highly parallel as noted above, computation at the horizon of a black hole is highly serial: every bit is essentially connected to every other bit over the course of a single logic operation. As noted above, the serial nature of computation at the black-hole limit can be deduced from the fact that black holes attain the Beckenstein bound $^{20-22}, k_{B} R E / \hbar c S=1 / 2 \pi$.

\section{(Box 2: Can a black hole compute?}

No information can escape from a classical black hole: what goes in does not come out. The quantum mechanical picture of a black hole is different, however. First of all, black holes are not quite black: they radiate at the Hawking temperature. $T$ given above. In addition, the well-known statement that 'a black hole has no hair'-i.e., from a distance all black holes with the same charge and angular momentum look essentially alike — is 
now known to be not always true ${ }^{91-93}$. Finally, recent work in string theory ${ }^{94-96}$ suggests that black holes do not actually destroy the information about how they were formed, but instead process it and emit the processed information as part of the Hawking radition as they evaporate: what does in does come out, but in an altered form.

If the latter picture is correct, then black holes could in principle be 'programmed': one forms a black hole whose initial conditions encode the information to be processed, lets that information be processed by the Planckian dynamics at the hole's horizon, and gets out the answer to the computation by examining the correlations in the Hawking radiation emitted when the hole evaporates. Despite our lack of knowledge of the precise details of what happens when a black hole forms and evaporates (a full account must await a more exact treatment using whatever theory of quantum gravity and matter turns out to be the correct one), we can still provide a rough estimate how much information is processed during this computation. Using Page's results on the rate of evaporation of a black hole ${ }^{88}$, we obtain a lifetime for the hole $t_{\text {life }}=G^{2} m^{3} / 3 C \hbar c^{4}$, where $C$ is a constant that is depends on the number of species of particles with a mass less than $k_{B} T$, where $T$ is the temperature of the hole. For $O\left(10^{1}-10^{2}\right)$ such species, $C$ is on the order of $10^{-3}-10^{-2}$,

leading to a lifetime for a 1 kilogram black hole of $\approx 10^{-19}$ seconds, during which time the hole can perform $\approx 10^{32}$ operations on its $\approx 10^{16}$ bits. As the actual number of effectively massless particles at the Hawking temperature of a one-kilogram black hole is likely to be considerably larger than $10^{2}$, this number should be regarded as an upper bound on the actual number of operations that could be performed by the hole. Interestingly, although this hypothetical computation is performed at ultra-high densities and speeds, the total number of bits available to be processed is not far from the number available to current computers operating in more familiar surroundings. )

\section{Constructing ultimate computers}

Throughout this entire discussion of the physical limits to computation, no mention has been made of how to construct a computer that operates at those limits. In fact, contemporary quantum 'microcomputers' such as those constructed using nuclear magnetic resonance ${ }^{76-80}$ do indeed operate at the limits of speed and memory space described above. Information is stored on nuclear spins, with one spin registering one bit. The time it takes a bit to flip from a state $|\uparrow\rangle$ to an orthogonal state $|\downarrow\rangle$ is given by $\pi \hbar / 2 \mu B=\pi \hbar / 2 E$, where $\mu$ is the spin's magnetic moment, $B$ is the magnetic field, and $E=\mu B$ is the average energy of interaction between the spin and the magnetic field. To perform a quantum logic operation between two spins takes a time $\pi \hbar / 2 E_{\gamma}$, where $E_{\gamma}$ is the energy of interaction between the two spins. 
Although NMR quantum computers already operate at the limits to computation set by physics, they are nonetheless much slower and process much less information than the ultimate laptop described above. This is because their energy is largely locked up in mass, thereby limiting both their speed and their memory. Unlocking this energy is of course possible, as a thermonuclear explosion indicates. Controlling such an 'unlocked' system is another question, however. In discussing the computational power of physical systems in which all energy is put to use, we assumed that such control is possible in principle, although it is certainly not possible in current practice. All current designs for quantum computers operate at low energy levels and temperatures, exactly so that precise control can be exerted on their parts.

As the discussion of error correction above indicates, the rate at which errors can be detected and rejected to the environment by error correction routines puts a fundamental limit on the rate at which errors can be committed. Suppose that each logical operation performed by the ultimate computer has a probability $\epsilon$ of being erroneous. The total number of errors committed by the ultimate computer per second is then $2 \epsilon E / \pi \hbar$. The maximum rate at which information can be rejected to the environment is, up to a geometric factor, $\ln 2 c S / R$ (all bits in the computer moving outward at the speed of light). Accordingly, the maximum error rate that the ultimate computer can tolerate is $\epsilon \leq \pi \ln 2 \hbar c S / 2 E R=2 t_{\text {flip }} / t_{\text {com }}$. That is, the maximum error rate that can be tolerated by the ultimate computer is the inverse of its degree of parallelization.

Suppose that control of highly energetic systems were to become possible. Then how might these systems be made to compute? As an example of a 'computation' that might be performed at extreme conditions, consider a heavy-ion collision that takes place in heavyion collider at Brookhaven ${ }^{97}$. If one collides 100 on 100 nucleons at $200 \mathrm{GeV}$ per nucleon, the operation time is $\pi \hbar / 2 E \approx 10^{-29}$ seconds. The maximum entropy can be estimated to be approximately to be 4 per relativistic pion (to within a factor of less than 2 associated with the overall production rate per mesons) of which there are approximately $10^{4}$ per central collision in which only a few tens of nucleons are spectators. Accordingly, the total amount of memory space available is $S / k_{B} \ln 2 \approx 10^{4}-10^{5}$ bits. The collision time is short: in the center of mass frame the two nuclei are Lorentz contracted to $D / \gamma$ where $D=12-13$ fermi and $\gamma=100$, giving a total collision time of $\approx 10^{-25}$ seconds. During the collision, then, there is time to perform approximately $10^{4}$ operations on $10^{4}$ bits a relatively simple computation. (The fact that only one operation per bit is performed suggests that there is insufficient time to reach thermal equilibrium, an observation that is confirmed by detailed simulations.) The heavy ion system could be programmed by manipulating and preparing the initial momenta and internal nuclear states of the ions. 
Of course, one does not expect to be able do word processing on such a 'computer.' Rather one expects to uncover basic knowledge about nuclear collisions and quark-gluon plasmas: in the words of Heinz Pagels, the plasma 'computes itself.'98

At the greater extremes of a black hole computer, we assumed that whatever theory (string theory, $\mathrm{M}$ theory?) turns out to be the correct theory of quantum matter and gravity, it is possible to prepare initial states of such systems that causes their natural time evolution to carry out a computation. What assurance do we have that such preparations exist, even in principle?

Physical systems that can be programmed to perform arbitrary digital computations are called computationally universal. Although computational universality might at first seem to be a stringent demand on a physical system, a wide variety of physical systems - ranging from nearest neighbor Ising models ${ }^{52}$ to quantum electrodynamics ${ }^{84}$ and conformal field theories ${ }^{86}$ — are known to be computationally universal ${ }^{51-53,55-65}$. Indeed, computational universality seems to be the rule rather than the exception. Essentially any quantum system that admits controllable nonlinear interactions can be shown to be computationally universal ${ }^{60-61}$. For example, the ordinary electrostatic interaction between two charged particles can be used to perform universal quantum logic operations between two quantum bits. A bit is registered by the presence or absence of a particle in a mode. The strength of the interaction between the particles, $e^{2} / r$, determines the amount of time $t_{f l i p}=\pi \hbar r / 2 e^{2}$ it takes to perform a quantum logic operation such as a Controlled-NOT on the two particles. Interestingly, the time it takes to perform such an operation divided by the amount of time it takes to send a signal at the speed of light between the bits $t_{\text {com }}=r / c$ is a universal constant, $t_{f l i p} / t_{\text {com }}=\pi \hbar c / 2 e^{2}=\pi / 2 \alpha$, where $\alpha=e^{2} / \hbar c \approx 1 / 137$ is the fine structure constant. This example shows the degree to which the laws of physics and the limits to computation are entwined.

In addition to the theoretical evidence that most systems are computationally universal, the computer on which I am writing this article provides strong experimental evidence that whatever the correct underlying theory of physics is, it supports universal computation. Whether or not it is possible to make computation take place in the extreme regimes envisaged in this paper is an open question. The answer to this question lies in future technological development, which is difficult to predict. If, as seems highly unlikely, it is possible to extrapolate the exponential progress of Moore's law into the future, then it will only take two hundred and fifty years to make up the forty orders of magnitude in performance between current computers that perform $10^{10}$ operations per second on $10^{10}$ bits and our one kilogram ultimate laptop that performs $10^{51}$ operations per second on $10^{31}$ bits. 


\section{References}

1. Maxwell, J.C., Theory of Heat, (Appleton, London), 1871.

2. Smoluchowski, M. von, Z. Physik, 13, 1069 (1912).

3. Szilard, L., Z. Physik 53, 840-856 (1929).

4. Brillouin, L., 'Science and Information Theory,' (Academic Press, New York), 1953.

5. Landauer, R., IBM J. Res. Develop. 5, 183 (1961).

6. Keyes, R.W., Landauer, R., IBM Journal of Research and Development, 14, no. 2, 152-157 (1970).

7. Landauer, R., Nature, 335, 779-784 (1988).

8. Landauer, R., Physics Today, May 1991, 23-29 (1991).

9. Landauer, R., Phys. Lett. A, 217, 188-193 (1996).

10. von Neumann, J., 'Theory of Self-Reproducing Automata,' Lecture 3 (University of Illinois Press, Urbana), 1966.

11. Lebedev, D.S., Levitin, L.B., Information and Control 9, 1-22 (1966).

12. Levitin, L.B., in 'Proceedings of the 3rd International Symposium on Radio Electronics,' part 3, 1-15 (Varna, Bulgaria), 1970.

13. L.B. Levitin, Int. J. Theor. Phys. 21, 299-309 (1982).

14. Levitin, L.B. Physica D 120, 162-167 (1998).

15. Margolus, N., Levitin, L.B., in PhysComp96, T. Toffoli, M. Biafore, J. Leao, eds. (NECSI, Boston) 1996.

16. Margolus, N., Levitin, L.B., Physica D 120, 188-195 (1998).

17. Bremermann, H.J., in 'Self-Organizing Systems,' M.C. Yovits, G.T. Jacobi, and G.D. Goldstein, eds. (Spartan Books, Washington, D.C.), 1962.

18. Bremermann, H.J., in 'Proceedings of the Fifth Berkeley Symposium on Mathematical Statistics and Probability,' (University of California Press, Berkeley), 1967.

19. Bremermann, H.J., Int. J. Theor. Phys. 21, 203-217 (1982).

20. Bekenstein, J.D., Phys. Rev. D. 23, 287 (1981).

21. Bekenstein, J.D., Phys. Rev. Letters 46, 623 (1981).

22. Bekenstein, J.D., Phys. Rev. D. 30, 1669-1679 (1984).

23. Aharonov, Y., Bohm, D., Phys. Rev. 122, 1649 (1961).

24. Aharonov, Y., Bohm, D., Phys. Rev. B. 134 122, 1417 (1964).

25. Anandan, J., Aharonov, Y. Phys. Rev. Lett. 65, 1697 (1990).

26. Peres, A., Quantum Theory: Concepts and Methods, (Kluwer, Hingham) 1995.

27. Lecerf, Y. Comptes Rendus 257, 2597 (1963).

28. Bennett, C.H., IBM J. Res. Develop. 17, 525 (1973) 
29. Bennett, C.H., Int. J. Theor. Phys. 21, 905 (1982).

30. Bennett, C.H., Sci. Am. 257, 108 (1987).

31. Fredkin, E., Toffoli, T., Int. J. Theor. Phys. 21, 219 (1982).

32. K.K. Likharev Int. J. Theor. Phys., 21, 311-325 (1982).

33. Seitz, C.L. et al., in Proceedings of the 1985 Chapel Hill Conference on VLSI, (Computer Science Press) 1985.

34. Merkle, R.C., 'Reversible Electronic Logic Using Switches,' submitted to Nanotechnology, 1992.

35. Younis, S.G., Knight, T.F., in Proceedings of the 1993 Symposium on Integrated Systems, Seattle, Washington, (MIT Press, Cambridge) 1993.

36. Lloyd, S., Pagels, H. Ann. Phys. 188, 186-213, (1988).

37. Lloyd, S., Phys. Rev. A 39, 5378-5386, (1989).

38. Zurek, W.H., Nature 341, 119 (1989).

39. Leff, H.S., Rex, A.F., Maxwell's Demon: Entropy, Information, Computing, Princeton University Press, Princeton, 1990.

40. Lloyd, S., Phys. Rev. A 56, 3374-3382, (1997).

41. Benioff, P., J. Stat. Phys. 22, 563 (1980).

42. Benioff, P., Phys. Rev. Lett. 48, 1581 (1982).

43. Feynman, R.P., Int. J. Theor. Phys. 21, 467 (1982).

44. Feynman, R.P., Optics News 11, 11 (1985); reprinted in Found. Phys. 16, 507 (1986).

45. Zurek, W.H., Phys. Rev. Lett. 53, 391 (1984).

46. Peres, A., Phys. Rev. A 32, 3266 (1985).

47. Deutsch, D., Proc. Roy. Soc. Lond. A 400, 97 (1985).

48. Margolus, N., Ann. N.Y. Acad. Sci. 480, 487 (1986).

49. Deutsch, D., Proc. Roy. Soc. Lond. A 425, 73 (1989).

50. Margolus, N., Complexity, Entropy, and the Physics of Information, Santa Fe Institute Studies in the Sciences of Complexity VIII, W.H. Zurek, ed., (Addison Wesley, Redwood City, 1991) pp. 273-288.

51. Lloyd, S., Phys. Rev. Lett. 71, 943 (1993).

52. Lloyd, S., Science 261, 1569-1571 (1993).

53. Lloyd, S., J. Mod. Opt. 41, 2503 (1994).

54. Shor, P., in Proceedings of the 35th Annual Symposium on Foundations of Computer Science, S. Goldwasser, Ed., IEEE Computer Society, Los Alamitos, CA, 1994, pp. 124-134.

55. Lloyd, S., Sci. Am. 273, 140 (1995).

56. Divincenzo, D., Science 270, 255 (1995). 
57. DiVincenzo, D.P., Phys. Rev. A 51, 1015-1022 (1995).

58. Sleator, T., Weinfurter, H., Phys. Rev. Lett. 74, 4087-4090 (1995).

59. Barenco, A., Bennett, C.H., Cleve, R., DiVincenzo, D.P., Margolus, N., Shor, P., Sleator, T., Smolin, J.A., Weinfurter, H., Phys. Rev. A 52, 3457-3467 (1995).

60. Lloyd, S., Phys. Rev. Lett., 75, 346-349 (1995).

61. Deutsch, D., Barenco, A., Ekert, A., Proc. Roy. Soc. A 449, 669-677 (1995).

62. Cirac, J.I., Zoller, P. Phys. Rev. Lett. 74, 4091-4094 (1995).

63. Pellizzari, T., Gardiner, S.A., Cirac, J.I., Zoller, P., Phys. Rev. Lett. 75, 3788-3791 (1995).

64. Turchette, Q.A., Hood, C.J., Lange, W., Mabuchi, H., Kimble, H.J., Phys. Rev. Lett. 75, 4710-4713 (1995).

65. Monroe, C., Meekhof, D.M., King, B.E., Itano, W.M., Wineland, D.J., Phys. Rev. Lett. 75, 4714-4717 (1995).

66. Grover, L.K., in Proceedings of the 28th Annual ACM Symposium on the Theory of Computing, ACM Press, New York, 1996, pp. 212-218.

67. Lloyd, S., Science 273, 1073-1078 (1996).

68. Zalka, C., Proc. R. Soc. Lond A. Mat. 454, 313-322 (1998).

69. Shor, P.W., Phys. Rev. A 52, R2493-R2496 (1995).

70. Steane, A.M., Phys. Rev. Lett. 77, 793-797 (1996).

71. Laflamme, R., Miquel, C., Paz, J.P., Zurek, W.H., Phys. Rev. Lett. 77, 198-201 (1996).

72. DiVincenzo, D.P., Shor, P.W., Phys. Rev. Lett. 77, 3260-3263 (1996).

73. Shor, P., in Proceedings of the 37th Annual Symposium on the Foundations of Computer Science, IEEE Computer Society Press, Los Alamitos, 1996, pp. 56-65.

74. Preskill, J., Proc. Roy. Soc. Lond. Ser. A 454, 385 (1998).

75. Laflamme, R., Knill, M., Zurek, W.H., Science 279, 342 (1998).

76. Cory, D.G., Fahmy, A.F., Havel, T.F., in PhysComp96, Proceedings of the Fourth Workshop on Physics and Computation, T. Toffoli, M. Biafore, J. Leão, eds., (New England Complex Systems Institute, Boston) 1996.

77. Gershenfeld, N.A., Chuang, I.L. Science 275, 350-356 (1997).

78. Chuang, I.L., Vandersypen, L.M.K., Zhou, X., Leung, D.W., Lloyd, S., Nature 393, 143-146 (1998) May, 1998.

79. Jones, J.A., Mosca, M., Hansen, R.H., Nature 393, 344-346 (1998).

80. Chuang, I.L., Gershenfeld, N., Kubinec, M., Phys. Rev. Lett. 80, 3408-3411 (1998).

81. Kane, B., Nature 393, 133 (1998).

82. Nakamura, Y., Pashkin, Yu.A., Tsai, J.S., Nature 398, 305 (1999) 
83. Mooij, J.E., Orlando, T.P., Levitov, L., Tian, L., van der Wal, C.H., Lloyd, S., Science 285, 1036-1039 (1999).

84. Lloyd, S., Braunstein, S., Phys. Rev. Lett. 82, 1784-1787 (1999).

85. Abrams, D., Lloyd, S., Phys. Rev. Lett. 81, 3992-3995, (1998).

86. Freedman, M., unpublished.

87. Zel'dovich, Ya. B., Novikov, I.D., Relativistic Astrophysics, (Univ. of Chicago Press, Chicago), 1971.

88. Page, D.N., Phys. Rev. D 13, 198 (1976).

89. K.S. Thorne, R.H. Price, D.A. Macdonald, Black Holes: The Membrane Paradigm, (Yale, New Haven) 1986. In particular, chapter VIII, 'The Thermal Atmosphere of a Black Hole,' by K.S. Thorne, W.H. Zurek, and R.H. Price.

90. Novikov, I.D., Frolov, V.P., Black Holes, (Springer-Verlag, Berlin) 1986.

91. Coleman, S., Preskill, J., Wilczek, F., Phys. Rev. Lett. 67 1975-1978 (1991).

92. Preskill, J., Phys. Scr. T 36, 258-264 (1991).

93. Fiola, T.M., Preskill, J., Strominger A., Phys. Rev. D 50, 3987-4014 (1994).

94. Susskind, L., Uglum, J., Phys. Rev. D 50, 2700 (1994).

95. Strominger A., Vafa, C., Phys. Lett. B 37, 99 (1996).

96. Das, S.R., Mathur, S.D., Nucl. Phys. B 478, 561-576 (1996).

97. Sidney H. Kahana, private communication.

98. Pagels, H., The Cosmic Code: quantum physics as the language of nature, (Simon and Schuster, New York) 1982. 


\section{Figure 1: The Ultimate Laptop}

The 'ultimate laptop' is a computer with a mass of one kilogram and a volume of one liter, operating at the fundamental limits of speed and memory capacity fixed by physics. The ultimate laptop performs $2 m c^{2} / \pi \hbar=5.4258 \times 10^{50}$ logical operations per second on $\approx 10^{31}$ bits. Although its computational machinery is in fact in a highly specified physical state with zero entropy, while it performs a computation that uses all its resources of energy and memory space it appears to an outside observer to be in a thermal state at $\approx 10^{9}$ degrees Kelvin. The ultimate laptop looks like a small piece of the Big Bang.

\section{Figure 2: Computing at the Black-Hole Limit}

The rate at which the components of a computer can communicate is limited by the speed of light. In the ultimate laptop, each bit can flip $\approx 10^{19}$ times per second, while the time to communicate from one side of the one liter computer to the other is on the order of $10^{9}$ seconds: the ultimate laptop is highly parallel. The computation can be speeded up and made more serial by compressing the computer. But no computer can be compressed to smaller than its Schwarzschild radius without becoming a black hole. A one-kilogram computer that has been compressed to the black hole limit of $R_{S}=$ $2 G m / c^{2}=1.485 \times 10^{-27}$ meters can perform $5.4258 \times 10^{50}$ operations per second on its $I=4 \pi G m^{2} / \ln 2 \hbar c=3.827 \times 10^{16}$ bits. At the black-hole limit, computation is fully serial: the time it takes to flip a bit and the time it takes a signal to communicate around the horizon of the hole are the same. 